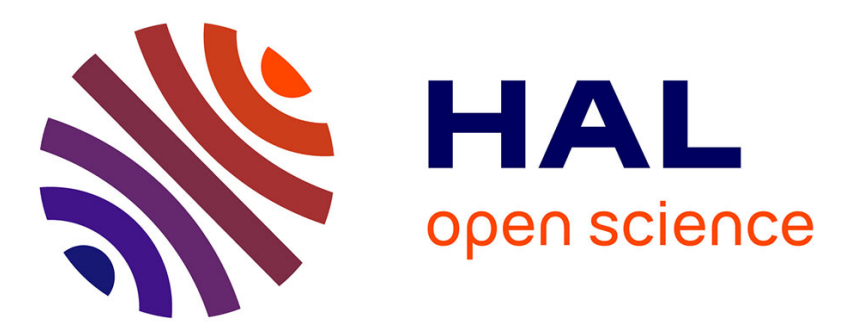

\title{
Nommer, couper, incorporer: Quand le nom rencontre le corps de l'image
}

\author{
Estelle Ingrand-Varenne
}

\section{To cite this version:}

Estelle Ingrand-Varenne. Nommer, couper, incorporer: Quand le nom rencontre le corps de l'image. Words/Les Mots 13th annual symposium of the International Medieval Society (IMS Paris), Jun 2016,

Paris, France. pp.209-228. halshs-03079190

\section{HAL Id: halshs-03079190 \\ https://shs.hal.science/halshs-03079190}

Submitted on 9 Feb 2022

HAL is a multi-disciplinary open access archive for the deposit and dissemination of scientific research documents, whether they are published or not. The documents may come from teaching and research institutions in France or abroad, or from public or private research centers.
L'archive ouverte pluridisciplinaire $\mathbf{H A L}$, est destinée au dépôt et à la diffusion de documents scientifiques de niveau recherche, publiés ou non, émanant des établissements d'enseignement et de recherche français ou étrangers, des laboratoires publics ou privés. 
Estelle Ingrand-Varenne, « Nommer, couper, incorporer : Quand le nom rencontre le corps de l'image ", Words in the Middle Ages/Les Mots an Moyen Age, V. Turner and V. Debiais dir., Turnhout : Brepols, 2020, p. 209-228.

Version auteur 


\section{Nommer, couper, incorporer : quand le nom rencontre le corps de l'image}

Parmi les nombreuses possibilités de cohabitation du mot et de l'image, la culture écrite médiévale, tant dans l'Occident latin que dans l'Orient byzantin, a développé une mise en scène particulière pour certains termes : le nom ou l'expression nominale, placé de chaque côté de la représentation d'un personnage ou d'une figure. En contexte épigraphique, cette mise en scène touche essentiellement les noms de personne appartenant à la sphère du sacré, la Vierge, les apôtres, les saints comme sur la baie 224 de la cathédrale de Bourges, ${ }^{1}$ mais aussi les noms des vices, des vertus, des mois et du zodiaque alors personnifiés. Elle n'est presque jamais utilisée pour les noms de contemporains.

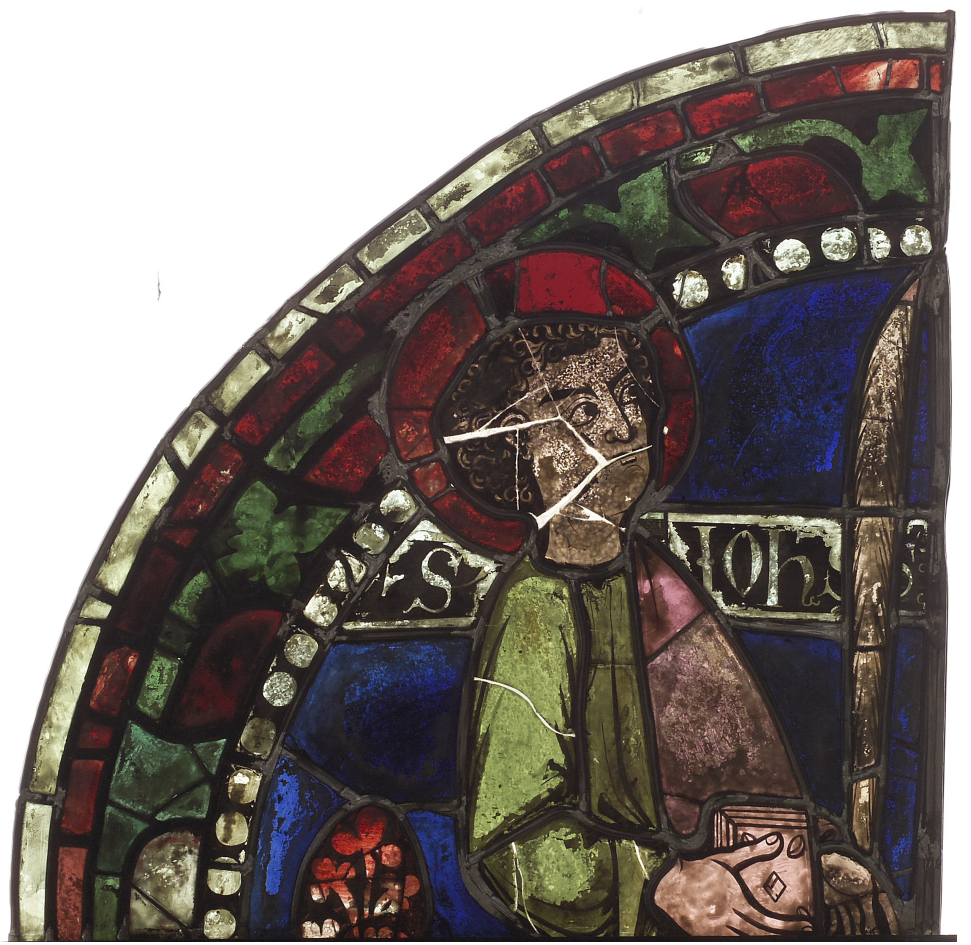

[Figure 1 : Détail de la rosace de saint Jean et de Jacob (baie 224), entre 1230 et 1255, cathédrale Saint-Étienne de Bourges. Cliché : C. Lemzaouda, Centre André Chastel (UMR 8150).]

Déjà expérimenté dans l'Antiquité, puis dans le monde manuscrit, cette mise en scène fleurit à nouveau entre la seconde moitié $\mathrm{du} \mathrm{XI}^{\mathrm{e}}$ et le $\mathrm{XIII}^{\mathrm{e}}$ dans les arts précieux et monumentaux. ${ }^{2}$ L'étude des 26 volumes du Corpus des inscriptions de la France médiévale montre que trois techniques l'emploient particulièrement, quoique de manière plus ou moins variable selon les siècles : l'orfèvrerie, la peinture murale, le vitrail, et qu'elle concerne surtout les images plates ou à faible relief. La sculpture n'est mobilisée que dans une moindre mesure, ${ }^{3}$ puisque c'est l'époque où se développe la statuaire, c'est-à-dire des formes détachées du fond ; or, c'est sur ce fond que se trouve inscrit le nom. ${ }^{4}$

\footnotetext{
${ }^{1}$ Corpus des inscriptions de la France médiévale, désormais CIFM, 26, 61, p. 87-88.

${ }^{2}$ Cela ne signifie pas qu'elle n'a plus cours au bas Moyen Âge, mais sa fréquence est moindre. C'est pourquoi notre étude se focalisera sur les XI $\mathrm{e}^{\mathrm{e}}$-XIII ${ }^{\mathrm{e}}$ siècles.

${ }^{3}$ Par exemple, les noms de Jean et Barthélémy de chaque côté de leur tête sur un chapiteau conservé au Musée de Valence, du XI'-XII' s. (CIFM, 16, Drôme 78, p. 197-198, pl. LXIV, fig. 129-130).

${ }^{4}$ Jean-Claude Bonne définit le fond comme "le lieu affinitaire de toutes choses, le terrain qui contrôle leur association ou leur affrontement", tout en notant qu'il y a une différence d'appréhension du fond entre la
} 
Cette manière de placer le nom est si courante qu'elle en est devenue anodine. Christian Heck ne la mentionne pas dans sa "typologie du texte court dans l'image", certes non exhaustive et basée sur des exemples manuscrits. ${ }^{5}$ Elle n'en est pas moins déroutante, par le lien qu'elle tisse entre le visuel et le textuel grâce à une division et une incorporation. Les études récentes sur les formes de communication non-verbales des inscriptions, ${ }^{6}$ les interactions entre texte et image, ${ }^{7}$ les rapports proxémiques des noms comme révélateur de sens, ${ }^{8}$ incitent à analyser cette mise en scène de manière globale, car elle se place au cœur d'une constellation de questions touchant tant à l'histoire de l'écriture et la linguistique, qu'à l'iconographie, la sémiotique et l'anthropologie.

Ce sont ces implications sémantiques, le réseau de sens qui se trame dans cette économie graphique, que cette étude se propose d'analyser à travers les trois verbes qui en donnent la recette : nommer, couper et incorporer, après quelques réflexions terminologiques en guise d'amuse-bouche.

\section{Comment nommer ce phénomène ?}

Deux verbes sont habituellement utilisés pour décrire cette disposition du nom distribué de part et d'autre de l'image : "flanquer" et "accoster". Tous deux renvoient à une localisation, un positionnement, un rapport topographique entre une chose qui est déjà là dans notre cas, l'image - et une autre qui est ajoutée - le nom -. Par son étymologie, "flanquer" évoque également le corps. Le flanc désigne en effet chez l'être humain chacune des deux parties latérales symétriques (droite et gauche) situées au-dessous des fausses côtes, et de façon générale la partie latérale du tronc, de tout le corps ; ce terme s'applique ensuite par analogie aux choses ou aux éléments. Nous verrons qu'il n'est finalement pas si approprié pour décrire précisément la localisation du nom par rapport au corps du personnage représenté, car celui-ci n'est pas au niveau du tronc. L'étymologie d' "accoster" est en ce sens plus neutre et renvoie simplement au "côté", quel qu'il soit. Remarquons également que ces verbes sont souvent employés à la forme passive, mettant ainsi au centre l'image, qui est sujet $\mathrm{du}$ verbe et qui devient le repère à partir duquel on s'oriente.

Pour qualifier ce phénomène dans leurs recherches ou dans l'édition des inscriptions qui demande une localisation précise des textes, les épigraphistes français ont choisi il y a une

sculpture romane et la sculpture gothique. J.-C. BonNE, L'art roman de face et de profil. Le tympan de Conques (Paris, 1984), p. 145.

${ }^{5}$ C. HECK, "Un nouveau statut pour la parole ? L'image légendée entre énoncé, commentaire, et parole émise", in Qu'est-ce que nommer? L'image légendée entre monde monastique et pensée scolastique, ed. C. HECK (Turnhout, 2010), pp. 7-28. Les huit catégories retenues pour sa typologie du texte court dans l'image selon l'emplacement et la disposition du texte sont: 1) Inscription immédiatement à l'extérieur de l'image, 2) Inscription dans l'encadrement de l'image, 3) Inscription librement déployée dans une partie de la composition, 4) Inscription matérialisant un texte sur un livre représenté dans l'image, 5) Inscription sur un cartel, un panonceau, 6) Le cartouche et le phylactère, 7) Un genre hybride : le schéma didactique, 8) Le traité dialogué.

${ }^{6}$ Voir les récents ouvrages collectifs : Viewing Inscriptions in the Late Antique and Medieval World, ed. A. EASTMOND (Cambridge, 2015); Writting matters: Presenting and Perceiving Monumental Inscriptions in Antiquity and the MiddleAges, ed. I. BERTI et al. (Berlin, 2017).

${ }^{7}$ L'étude de référence dans ce domaine est désormais l'ouvrage de V. DEBIAIS, La croisée des signes: L'écriture et les images médiévales (800-1200) (Paris, 2017).

${ }^{8}$ Voir l'analyse du placement des noms sur les reliquaires par B. HosteTLER : "The Iconography of Text: The Placement of an Inscription on a Middle Byzantine Reliquary", Eastern Christian Art 8 (2011), pp. 49-55 ; “The Limburg Staurotheke: A Reassessment", Anthanor 30 (2012), pp. 7-13; "Towards a Typology for the Placement of Names on Works of Art", in Inscribing Texts in Byzantium: Continuity, Invention, Transformation. Proceedings of the 49th Spring Symposium of Byzantine Studies, ed. I. TOTH et al. (sous presse). 
dizaine d'années un autre terme. C'est vers le vocabulaire poétique qu'ils se sont tournés, plus précisément la métrique, en empruntant le terme de "césure". ${ }^{9}$ Ainsi, dans l'édition des noms incisés sur la plaque de reliquaire de la Vraie Croix (1155-1165) conservée au Musée Dobrée à Nantes, les inscriptions sont dites "en césure", notamment Sera/phim de chaque côté des ailes repliées. ${ }^{10} \mathrm{Ce}$ choix, plutôt que d'autres mots comme "coupure", "division" ou "scission", permet de désigner - comme en poésie - une coupe qui est une suspension temporaire, un repos dans une continuité graphique voire iconographique. Ce changement de mot est aussi un changement de regard, qui déplace la focalisation portée sur l'image, avec les verbes "flanquer" et "accoster", sur celle du texte. Si, de fait, l'image est bien première dans le processus de réalisation artistique et le nom ajouté après, le résultat produit quant à lui ne crée ni hiérarchie, ni succession temporelle, il faut donc traiter les deux dans un même temps.

L'ensemble des relations évoquées par ces termes (le corps, le placement, la coupure) est à prendre en compte. Pour continuer cette recherche terminologique, nous proposons de parler de ce phénomène comme un dispositif, reprenant un terme développé par Michel Foucault et expliqué notamment par Daniel Russo. La définition de ce dernier est la suivante :

concept qui permet de comprendre les relations qui sont instaurées par l'écriture des mots sur des images [...] à l'intérieur d'un système graphique historiquement situé et au cœur duquel le support choisi, la matière utilisée, la technique de travail, apparaissent aussi comme des moyens importants. ${ }^{11}$

C'est bien ce qui est en jeu ici.

\section{Nommer}

Le processus de nomination au cœur de ce dispositif n'est pas celui de la nomination première, ex nihilo, mais le fait d'indiquer le nom d'un personnage déjà connu comme tel auprès de sa représentation. ${ }^{12}$ La présence de ce mot vient lever l'ambiguïté et la confusion potentielle, fixer le sens et réduire le champ des possibles, car le nom (propre) est un lieu de mémoire et un organisateur. ${ }^{13}$ Comme l'a expliqué Roland Barthes, le nom dans l'image, en tant que message linguistique, a une fonction d'ancrage et de relais par rapport au message iconique. ${ }^{14} \mathrm{Ce}$ que Vincent Debiais dit de l'image médiévale en apportant un élément supplémentaire: "la nomination et la désignation graphique par le nom accordent une existence articulée de la figure au monde" ; il propose de voir le nom non seulement comme

\footnotetext{
${ }^{9}$ Le terme de "césure" apparaît dans le volume 23 du CIFM (par exemple dans les notices n $68,69,134$ ) paru en 2008, réalisé par la nouvelle équipe en charge du Corpus, notamment Vincent Debiais. La paternité de cette expression lui revient.

${ }^{10}$ CIFM 23, 68, p. 78-79.

${ }^{11}$ D. Russo, "Des lettres sur l'image dans l'art du Moyen Âge. Pour une nouvelle articulation du textuel et du visuel", in Qu'est-ce que nommer?, p. 129. Voir aussi : G. AGAMBEN, Qu'est-ce qu'un dispositif ? (Paris, 2007); G. Agamben se propose de tracer une généalogie sommaire de ce terme d'abord à l'intérieur de l'œuvre de Foucault, puis dans un contexte historique plus ample.

${ }^{12}$ La bibliographie sur le nom, notamment le nom propre, est vaste et variée suivant l'approche choisie: anthropologique, linguistique, cognitive, historique... Nous nous focaliserons sur les études liant nom et image. Pour une bibliographie relativement complète du nom au Moyen Âge, voir le projet MEDNAME sur l'étude du nom dans la culture écrite médiévale (PHC Van Gogh, entre le CESCM Université de Poitiers et l'Université d'Utrecht, Pays-Bas).

${ }^{13}$ L'anonymat d'une représentation au contraire, comme l'a montré Henry Maguire pour l'art byzantin, pouvait augmenter son "opérativité" parce qu'on invoquait alors plusieurs saints. H. MAGUIRE, "Eufrasius and friends. On Names and Their Absence in Byzantine Art", in Art and Text in Byzantine Culture, ed. L. James (Cambridge, 2007), pp. 139-160.

${ }^{14}$ R. BARTHES, "Rhétorique de l'image", Communications 4 (1964,) p. 44.
} 
une distinction, ${ }^{15}$ mais aussi comme une "double articulation" avec le créateur et le reste des créatures. ${ }^{16}$ Ces notions de fixation et de démarcation, d'imbrication et de continuum seront à prendre en compte pour comprendre cette mise en scène en césure.

Les noms touchés par ce dispositif sont en premier lieu ceux des personnages de l'histoire biblique : Adam, les prophètes, tel Osée sur un médaillon émaillé au Musée de Rouen datant du début du XII ${ }^{\mathrm{e}}$ s., ${ }^{17}$ le roi Nabuchodonosor sculpté sur la frise sur le Mystère du Salut à la façade de Notre-Dame-la-Grande à Poitiers (Vienne, premier quart du XII ${ }^{\mathrm{e}} \mathrm{s}$.), ${ }^{18}$ les anges, la Vierge Marie et les apôtres.

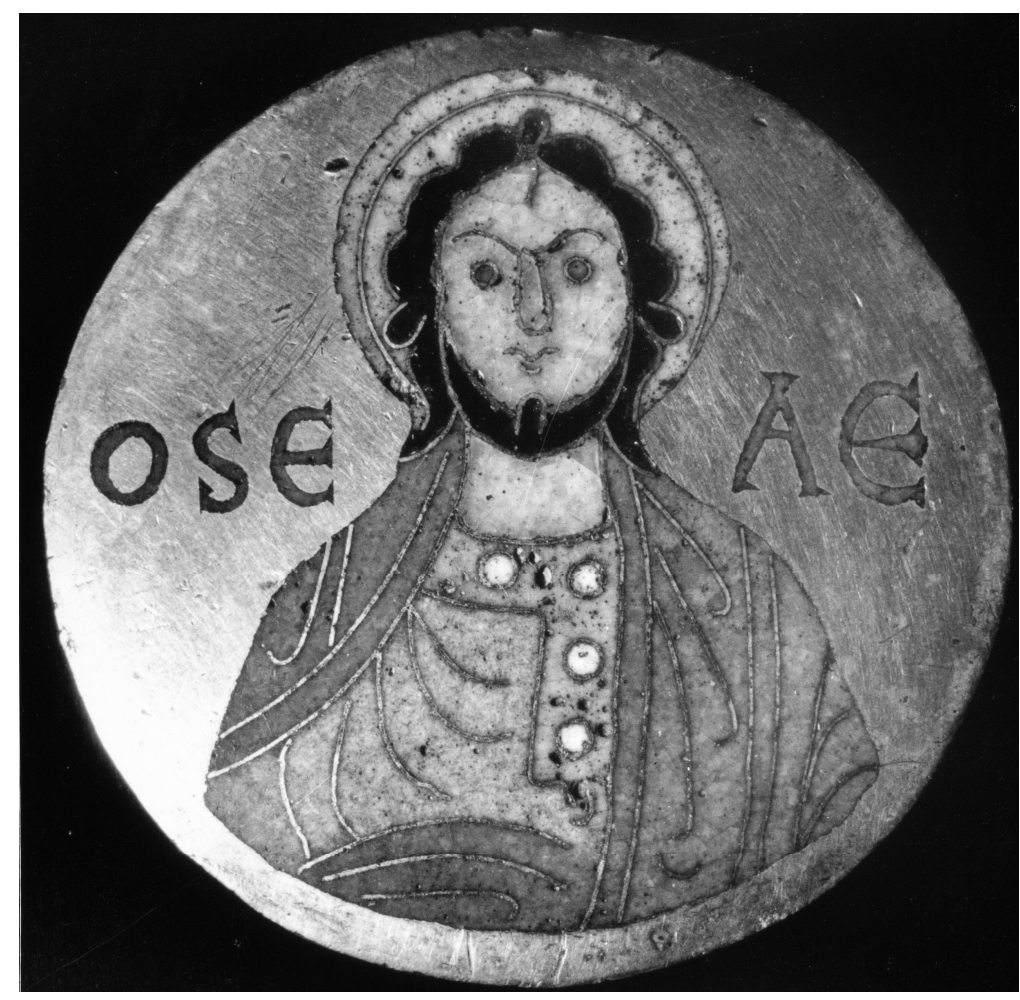

[Figure 2: Médaillon émaillé en bleu lapis représentant le prophète Osée, premier quart du XII ${ }^{e}$ s., Musée des Antiquités de Rouen (Seine-Maritime). Cliché : Jean Michaud/CIFM-CESCM.]

Les saints et les saintes sont la deuxième catégorie de personnage ainsi nommés. Il ne faudrait cependant pas réduire cette mise en scène aux noms propres et à la sphère du sacré. Le cycle de la Résurrection peint sur cinq registres dans le transept nord de l'église Saint-Sernin à Toulouse à la fin du XII ${ }^{e} s$. montre au premier et au deuxième registre des noms génériques de personnage : mili[te]/s cus/tos écrits de chaque côté des boucliers des soldats chargés de garder le tombeau; [mu]lyier/is de chaque côté d'une des saintes femmes rencontrant l'ange devant le tombeau vide. ${ }^{19}$ Le nom d'un donateur (frère/Ja[qu]es Le Cla/vier) est peint sur deux lignes en lettres rouges puis blanches de part et d'autre de sa figuration, agenouillée et les mains jointes, sur le vitrail placé aujourd'hui dans l'église de Hautot-sur-Seine (Seine-

\footnotetext{
${ }^{15}$ Les recherches des anthropologues sur le nom propre ont effectivement montré que celui-ci avait une fonction distinctive et classificatrice, et en même temps qu'il s'agissait d'une marque individuelle faite à des fins collectives. Voir L'écriture du nom propre, ed. A.-M. CHRISTIN (Paris, 1998), particulièrement la présentation faite par Anne-Marie Christin, pp. 7-8.

${ }^{16}$ DEBIAIS, La croisée des signes, p. 27.

${ }^{17}$ CIFM 22, 266, p. 341, fig. 124.

${ }^{18}$ CIFM I-1, 24, p. 22-25, pl. XIV, fig. 27, CIFM Hors Série II, 53 à paraître.

${ }^{19}$ CIFM 7, 14, p. 34-35, pl. II-III, fig. 3-6.
} 
Maritime, c. 1265). ${ }^{20}$ Des personnages négatifs peuvent également avoir leur nom mentionné avec cette mise en scène : Barvahim, bourreau de sainte Catherine d'Alexandrie, a son nom peint en jaune (Bar/vahim) alors qu'il brandit son épée, sur le vitrail de la cathédrale d'Angers dédié à cette sainte (Maine-et-Loire, vers 1190-1210). ${ }^{21}$

Une dernière catégorie de noms attire particulièrement l'attention : les noms communs désignant les vices ou les vertus, les mois du calendrier, les signes du zodiaque. Que janvier (Janu/ariu[s]) soit peint autour d'un personnage dans l'église de Lourouer-Saint-Laurent (Indre, début du XIII ${ }^{\mathrm{e}} \mathrm{s}$.) ${ }^{22}$ ou que Sagi/tarius et Aqu/arius soient gravés de chaque côté de la tête du sagittaire et du verseau sculptés sur des bas-reliefs conservés au Musée Gadagne à Lyon (XII ${ }^{\mathrm{e}} \mathrm{s}$.), ${ }^{23}$ montre sans doute que ces termes relèvent de l'allégorie, la personnification d'une idée abstraite, ${ }^{24}$ le nom commun devenant alors par antonomase un nom propre.

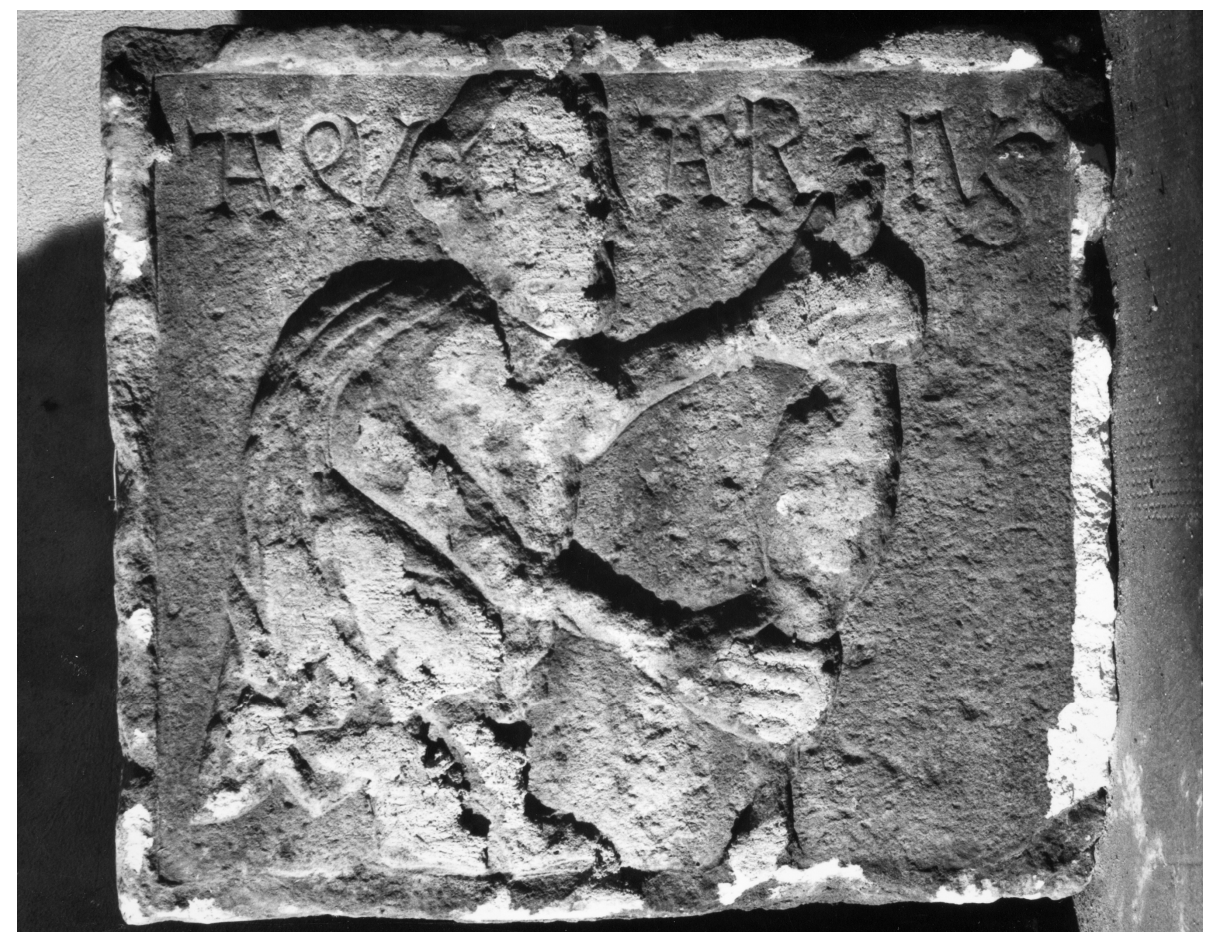

[Figure 3. : Bas-relief du verseau (XII s.), Musée Gadagne de Lyon (Rhône). Cliché : Jean Michaud/CIFM.]

Ce procédé est d'autant plus frappant avec les vertus, notamment la charité, qualifiée de sainte $(s(a n) c(t) a /$ Karitas $)$, alors qu'elle est représentée sous les traits d'une femme nimbée distribuant des aumônes sur un des chapiteaux du portail droit de l'église de Bourg-Argental

\footnotetext{
${ }^{20}$ CIFM 22, 167 bis, p. 254, fig. 89. Ce vitrail provient de l'ancienne commanderie de Sainte-Vaubourg au Valde-la-Haye près de Rouen.

${ }^{21}$ CIFM 24, 25, p. 46-48.

${ }^{22}$ CIFM 25, 27, p. 46-48. Pour d'autres mois, voir : CIFM 13, Gard 30, p. 39, pl. XIII, fig. 25 ; CIFM 13, Vaucluse 27, p. 135, pl. XLVI, fig. 95 ; CIFM 25, 68, p. 94.

${ }^{23}$ CIFM 17, Rhône 46, p. 105-106, pl. L-LIII, fig. 83-90. Les signes du poisson ( pi/scus) et du verseau () et le centaure chasseur (ve/nator) bénéficient aussi d'une mise en scène en césure. Ces bas-reliefs proviennent d'une chapelle de Sainte-Foy-lès-Lyon, seul vestige du château féodal.

${ }^{24}$ P. ZuMThor, Essai de poétique médiévale (Paris, 1972), p. 128 : "la personnification transforme un nom commun (...) en un nom autodéterminé qui renvoie à la manière d'un prénom, au sujet d'actions réelles". Pour une étude complète, voir A. STRUBEL et C. HECK, L'allégorie dans l'art du Moyen Âge. Formes et fonctions. Héritages, créations, mutations (Turnhout, 2011); ainsi que G. DAHAN, Lire la Bible au Moyen Âge. Essais d'herméneutique médiévale (Genève, 2009) : chapitre 8 consacré à "L'allégorie dans l'exégèse chrétienne de la Bible au Moyen Âge", pp. 283-317.
} 
(Loire, XII $\left.{ }^{\mathrm{e}} \mathrm{s}.\right){ }^{25}$ Ainsi ce dispositif n'est pas intrinsèquement lié à l'hiératisme. Il peut être employé aussi pour des raisons de variations esthétiques, à côté d'autres mises en page.

Comment le nom apparaît-il dans ce dispositif? Il figure isolé et non intégré dans une phrase, comme le nom de Moïse dans les peintures de l'église Saint-Martin de Vicq (Indre) au $\mathrm{XII}^{\mathrm{e}} \mathrm{s.}^{26}$ Le nom est d'ailleurs une des rares catégories de mot à pouvoir apparaître seul. Il participe néanmoins à une autre syntaxe, dont les articulations sont à définir. Au niveau graphique, il n'a pas de marque statutaire spécifique, c'est-à-dire de majuscule, dans l'écriture épigraphique puisque celle-ci est en capitale (au sens large du terme) au moins jusqu'au XIV ${ }^{\mathrm{e}}$ s., et ne crée pas de hiérarchie entre les lettres, à l'exception des abréviations et des jeux de lettres. Les images qui ont recours à cette césure sont en majorité des représentations de personnages en buste et en pied, figurés pour eux-mêmes, hors de tout contexte, tels Marie et Martial de chaque côté du Christ sur la châsse de Champagnat, vers $1150 .{ }^{27}$ Certes, on peut trouver le nom en césure au sein d'une scène narrative, tel le nom d'Adam dans la scène de la Tentation peinte dans la chapelle Saint-Jean-des-Vignes de Saint-Plancard (Haute-Garonne, seconde moitié $\mathrm{du} \mathrm{XI}^{\mathrm{e}} \mathrm{s}$.), mais on peut se demander si ce dispositif ne fait pas de ce personnage une entité autonome s'articulant avec la narration.

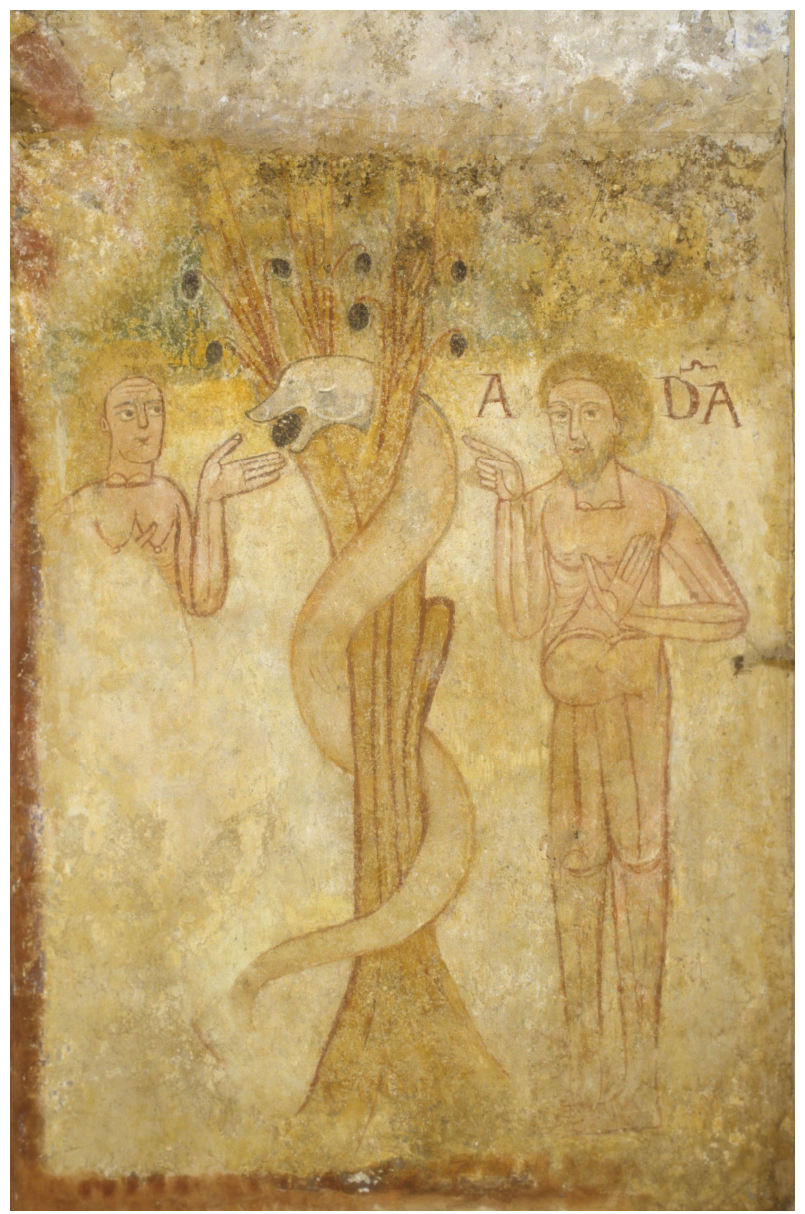

[Figure 4 : Peintures murales de la Tentation d'Adam et Ève, seconde moitié du XI s. shapelle Saint-Jean-desVignes de Saint-Plancard (Haute-Garonne). Cliché : Jean-Pierre Brouard /Base Romane-CESCM.]

\footnotetext{
${ }^{25}$ CIFM 18, Loire 4, p. 55-56, pl. XXVI, fig. 62-63. Pour d'autres inscriptions de vertus avec ce dispositif, voir CIFM 24, 210, p. 216 (la charité) ; CIFM 18, Haute-Loire 19, p. 104, pl. LI-LII, fig. 115-118 (la foi). ${ }^{26}$ CIFM 25, 35, p. 59-60.

${ }^{27}$ L'Euvre de Limoges. Émaux limousins du Moyen Âge, ed. É. Taburet-Delahaye et al. (Paris, 1995), nº10, pp. 90-92.
} 
La mention du nom aurait alors tendance à isoler le personnage, à le distinguer et à en faire une unité à part. Ce dispositif participe à un mouvement plus profond, car, comme l'a relevé Jean Wirth, le nom propre resurgit dans les arts monumentaux et précieux au moment où la narrativité décroit: l'interaction des personnages se raréfie au profit de leur simple juxtaposition ou de leur isolement. ${ }^{28}$

La réapparition de cette mise en scène dans les inscriptions est concomitante de l'"inflation du nom": les représentations et les noms de saints se multiplient, l'image du Christ, peu associée à son nom inscrit ou accompagnée de signes graphiques avant le $\mathrm{XI}^{\mathrm{e}} \mathrm{s}$., connaît le même changement, par exemple avec l'alpha et l'oméga. ${ }^{29}$ De même, on multiplie les attributs individuels, ces "accessoires servant à caractériser une figure", ${ }^{30}$ dans les images, de façon accélérée et systématique au début du XIII ${ }^{\mathrm{e}} \mathrm{s}$, , alors qu'un peu partout en Europe occidentale apparaissent des emblèmes nouveaux pour dire l'identité d'un individu et son appartenance à un groupe (noms patronymiques, vêtements, armoiries, sceaux). ${ }^{31}$ À côté des attributs, l'inscription du nom vient répondre à un besoin d'identification rapide de la figure, mais au-delà participe à son élaboration syntaxique et sémantique.

\section{Couper :}

La particularité de ce dispositif réside dans la coupure instaurée à l'intérieur de l'entité nominale. Elle interroge d'une part le nom, et plus largement le mot, en tant qu'unité graphique, d'autre part le procédé de division comme outil signifiant. C'est effectivement l'époque où, dans le monde manuscrit, on divise, on distingue, on segmente les œuvres en unités de sens avec des procédés visuels : livres, parties, chapitres, partie de chapitre, paragraphe, verset. ${ }^{32}$ Dans le même temps, on passe de la scriptio continua à l'écriture aérée, puis régulièrement séparée par des espacements. Au lieu de l'espace blanc, les inscriptions ont marqué les frontières des mots ou des séquences graphiques grâce à une interponctuation (un, deux, trois points verticaux) qui l'isole des autres éléments de la chaîne d'écriture : c'est entre le $\mathrm{XI}^{\mathrm{e}} \mathrm{s}$. et la première moitié du $\mathrm{XII}^{\mathrm{e}} \mathrm{s}$. qu'a lieu la véritable phase d'essai de ces points intercalaires, avant de se répandre dans la seconde moitié du XII ${ }^{\mathrm{e}}$ et au XIII ${ }^{\mathrm{e}} \mathrm{s}{ }^{33}$ Le mot est donc clairement pensé comme une unité d'écriture distincte, "atomique" si l'on veut; sa sécabilité a par conséquent d'autant plus d'importance. ${ }^{34}$

\footnotetext{
${ }^{28}$ J. WIRTH, “L'emprunt des propriétés du nom par l'image médiévale”, Études de lettres (1994), pp. 63-64. Les images deviennent des "unités discrètes" pour Jean Wirth.

${ }^{29}$ R. FAVREAU, "Des inscriptions pour l'image du Christ (XI ${ }^{\mathrm{e}}$-XIII ${ }^{\mathrm{e}}$ siècles)", in Qu'est-ce que nommer ?, pp. 169185.

${ }^{30}$ C. HECK, "Entre action et symbole : l'attribut héritage ou substitut de la scène narrative", in Des signes dans l'image. Usages et fonctions de l'attribut dans l'iconographie médiévale (du Concile de Nicée au Concile de Trente), ed. M. PASTOUREAU et al. (Turnhout, 2014), p. 45.

${ }^{31}$ M. Pastoureau, "Pour une histoire des attributs dans l'image médiévale", in Des signes dans l'image, pp. 11-34.

${ }^{32}$ D. POIREL, "Machina universatis : les mutations du texte aux XII $\mathrm{I}^{\mathrm{e}}-\mathrm{XIII}$ ' siècles", in Qu'est-ce que nommer ?, pp. 41-53.

33 E. IngRAnd-VARENNE, "Trois petits points: L"“interponctuation" dans les inscriptions médiévales", in Ponctuer l'œuvre médiévale : des signes au sens, ed. V. FASSEUR et al. (Genève, 2016), pp.215-232.

${ }^{34}$ Les délimitations externes des mots ont beaucoup intéressé les historiens et linguistes ces trente dernières années : à travers l'attention aux blanc, aux signes de ponctuation, et plus largement à la séquenciation de la chaîne écrite (les frontières des mots se perçoivent aussi grâce aux désinences et aux abréviations). Voir notamment : N. CATACH, "Les signes graphiques du mot à travers l'histoire", Langue française 119 (1998), pp. $10-23$.
} 
L'insertion d'un élément non alphabétique est la façon la plus commune de diviser un mot, particulièrement avec un élément iconographique. La rencontre du texte et de l'image, sous la forme d' "interférences" de l'image qui pénètre dans l'écriture, est courante au Moyen Âge central. Les éléments haut et bas transgressent la bordure et sortent du cadre : pointe du heaume ou de l'épée, extrémité des pattes et bout de la queue du cheval entrent dans le champ épigraphique des sceaux équestres, de même que les oiseaux et les lances dans les légendes de la Tapisserie de Bayeux, ou encore les pieds de saints dans leur nom peint sur les vitraux de Chartres. La rencontre se fait ici par l'extériorisation de l'image de son champ (même si celuici n'est pas toujours marqué et n'est jamais une clôture), pour intervenir dans le champ de l'écrit placé en périphérie.

Ces interférences ou coupures peuvent être aléatoires comme très significatives. Au linteau en marbre de Saint-Genis-des-Fontaines (1019/1020, Pyrénées-Orientales), on peut lire une phrase gravée sur deux lignes, de part et d'autre de la mandorle du Christ : + Anno videsimo quarto rennate Rot/berto rege Wilielmus gratia Dei aba. // Ista opera fieri jussit in onore $s(a n) c(t) I$ Gene/sii cenobii que vocant Fontanas). ${ }^{35}$

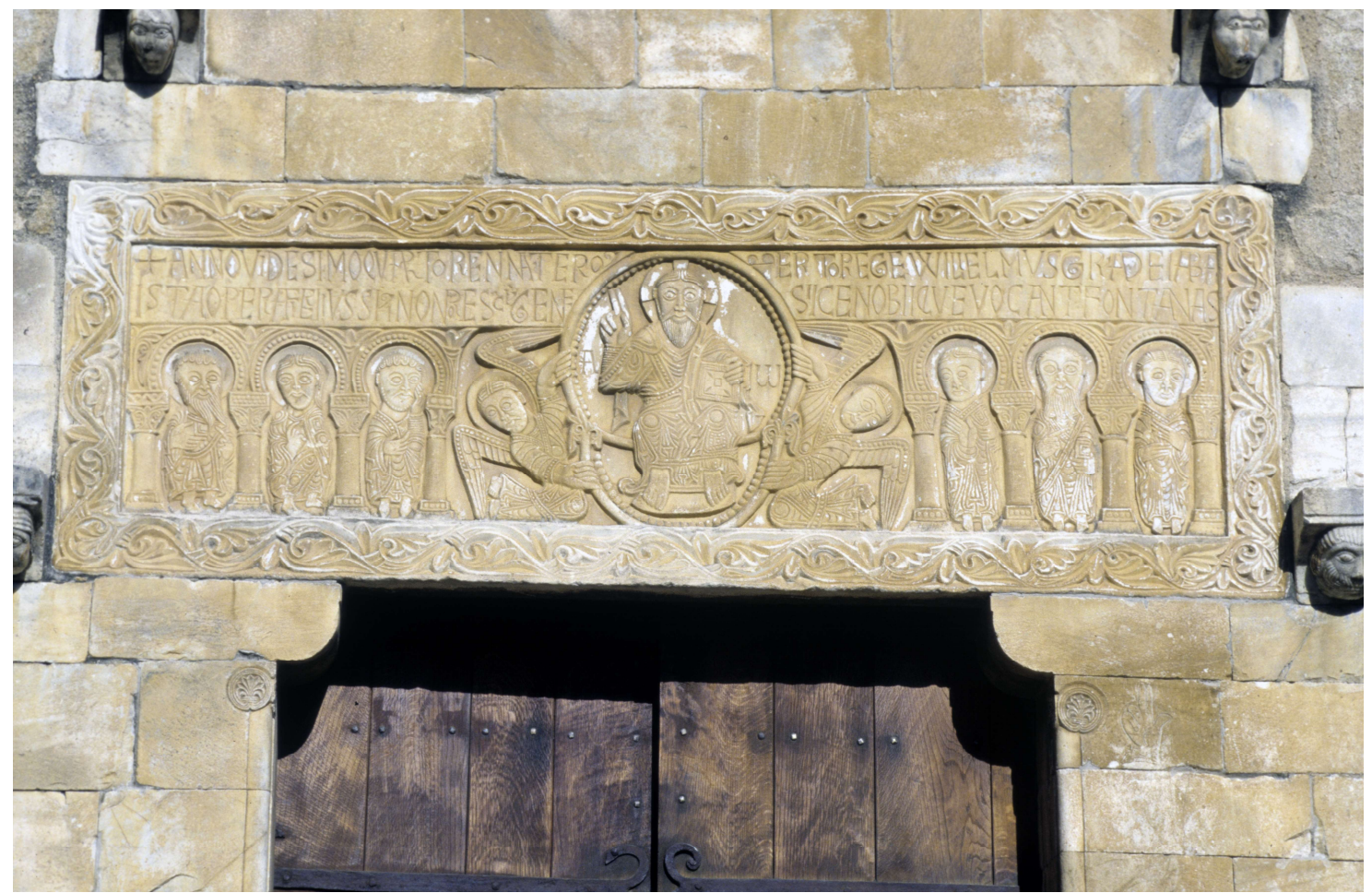

[Figure 5 : Linteau, gravé vers 1019/1020, à la façade occidentale de l'église Saint-Mchel de Saint-Génis-desFontaines (Pyrénées-Orientales). Cliché : Jean-Pierre Brouard/Bas Romane-CESCM]

Les noms du roi Robert et du saint titulaire de l'église sont placés au centre même du texte, l'un au-dessus de l'autre, et entrecoupés par la mandorle. Ce contact du nom gravé et de la représentation visuelle du Christ est à interpréter avec force. Le nom représentant la personne, on peut dire que Robert et Genès sont "touchés" par la sainteté du Christ, qu'ils "sont auprès"

\footnotetext{
${ }^{35}$ CIFM 11, Pyrénées-Orientales, 118, pp. 138-139, pl. LI, fig. 106 (traduction du CIFM : L'an vingt-quatrième du règne de Robert, Guillaume, abbé par la grâce de Dieu, ordonna de faire cette œuvre en l'honneur du monastère Saint-Genès qu'on appelle des Fontaines). Cette sculpture n'était pas originellement un linteau, mais un bas-relief; elle n'a été placée au-dessus de la porte de l'église qu'au XII ${ }^{\mathrm{e}} \mathrm{s}$. La bibliographie sur cette œuvre est riche, on verra l'article d'Olivier Poisson qui résume les différentes interprétations proposées par les historiens d'art: "Le linteau dans la façade : notes sur les portails de Saint-Genis-des-Fontaines et de SaintAndré (Roussillon)", Les Cahiers de Saint-Michel de Cuxa XLV (2014), pp. 197-210.
} 
de Dieu, tant matériellement que spirituellement. De même au tympan de Mervilliers (fin XII s., Eure-et-Loir) qui représente une scène de donation entre un chevalier et saint Georges, la croix de la couronne du Christ pénètre dans le champ épigraphique au milieu du nom du donateur, Renbautus, placé au milieu de l'arc du tympan. ${ }^{36}$ La différence entre les diverses exemples évoqués et le dispositif étudié est que celui-ci ne concerne qu'une seule et même entité, représentée à la fois par son nom et par son image.

Où couper le mot? La comparaison des différentes césures du nom de Marie montre que celle-ci occupe une place variable: SC/A MARIA, ${ }^{37}$ SCA/MARIA, ${ }^{38}$ S MA/RIA ou $\mathrm{MA} / \mathrm{RIA}^{39}$ (le cas le plus répandu, comme à Pouzauges); S MAR/IA, ${ }^{40} \mathrm{~S} / \mathrm{MA} \mathrm{RI} / \mathrm{A}{ }^{41}$

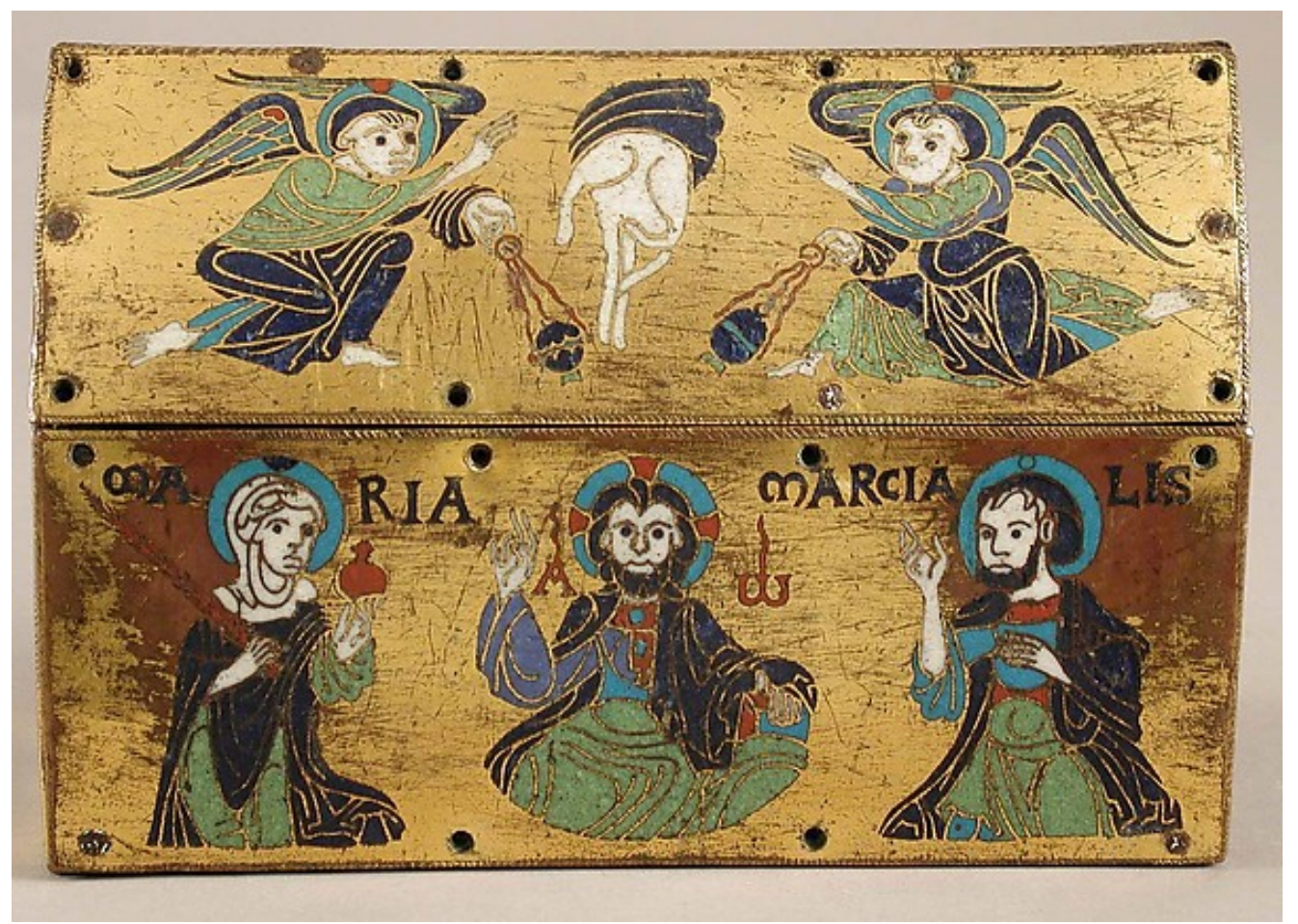

[Figure 6: Châsse émaillée provenant de l'église Saint-Martial de Champagnat (Creuse), réalisée vers 1150, conservée au Metropolitan Museum of Art à New-York. Cliché : Wikimedia common.]

Même si la répartition syllabique est récurrente dans ce cas, les coupures peuvent intervenir à n'importe quel endroit du nom. C'est davantage un équilibre graphique de chaque côté qui est recherché. Pour certaines expressions nominales composées de plusieurs mots, on a éprouvé le besoin de couper et répartir les deux termes. C'est le cas de l'inscription Agnus

\footnotetext{
${ }^{36}$ Voir l'étude de Cécile Voyer, qui fait le point sur l'historiographie et propose de nouvelles interprétations et datation de cet œuvre, le tympan est vu comme "une définition en image de la valeur efficace de l'acte diplomatique". C. VOYER, "Le geste efficace : le don du chevalier au saint sur le tympan de Mervilliers (XII" siècle)", in Chevalerie \& christianisme aux XII ${ }^{e}-X I I I^{e}$ siècles, ed. M. AURELL et al. (Rennes, 2011), pp.101-121.

37 CIFM 24, 196, pp. 205-206 (verrière de l'enfance et des miracles de la Vierge, à la cathédrale du Mans, $c$. 1235).

${ }^{38}$ CIFM 17, Isère 18, pp. 44-45, pl. XIV, fig. 29-31 (tympan de l'église Saint-Alban-du-Rhône, première moitié du XII ${ }^{\mathrm{e}}$ s.).

${ }^{39}$ CIFM II, Haute-Vienne 3, p. 91, pl. XX, fig. 39-40 (châsse émaillée de l'église de Bellac, c. 1200) ; CIFM 23, 134, pp. 132-133 (peintures murales de l'histoire de la Vierge dans l'église Notre-Dame de Pouzauges, Vendée) ; CIFM 19, Nièvre 16, pp. 41-43, pl. XIV-XVI (plaque de reliure au musée Blandin de Nevers, c. 1180) ; CIFM 18, Puy-de-Dôme 74, pp. 224-228, pl. CXX-CXXVI, fig. 257-270 (châsse de Saint-Calmine dans l'église SaintPierre de Mozac, fin XII s.) ; Euvre de Limoges 10, pp. 90-92, châsse de Saint-Martial de Champagnat (Creuse).

${ }^{40}$ Metropolitan Museum, Prov : Made in Meuse Valley, Netherlands, Rond en émail champlevé.

${ }^{41}$ Euvre de Limoges 1, pp. 68-70 (autel portatif émaillé de Conques).
} 
Dei peinte autour de l'Agneau dans la crypte de Notre-Dame-la-Grande à Poitiers à la fin du $\mathrm{XI}^{\mathrm{e}} \mathrm{s.}^{42}$ La césure n'est pas entre Agnus et Dei, mais à l'intérieur de chacun des deux mots, placés l'un au-dessous de l'autre ( $\mathrm{Ag} / \mathrm{nus} \mathrm{De} / \mathrm{i})$.

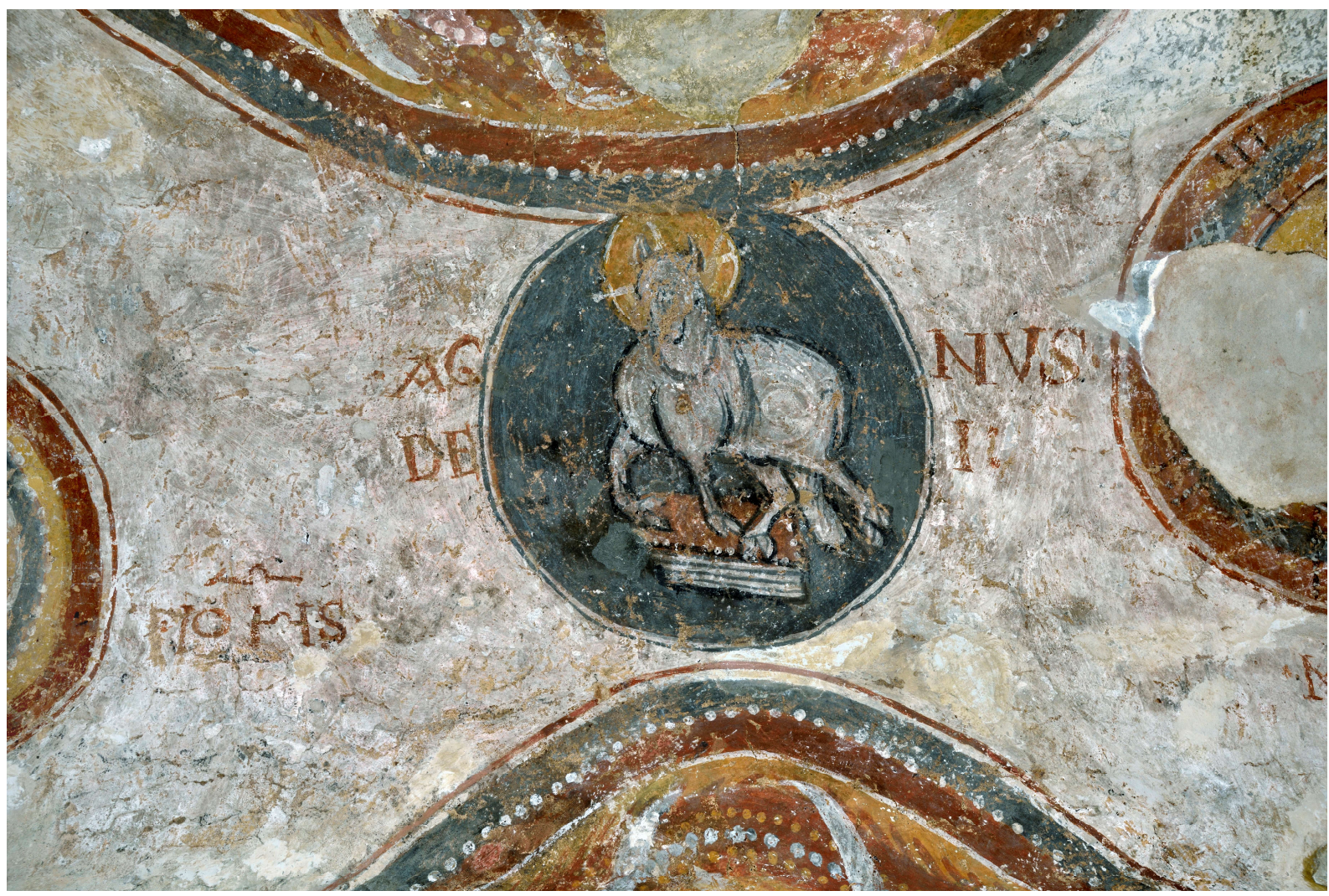

[Figure 7: Peintures murales de l'Agneau, fin du XI ${ }^{\mathrm{e}}$ s., crypte de Notre-Dame-la-Grande à Poitiers. Cliché : Jean-Pierre Brouard/CIFM-CESCM.]

Cet exemple permet d'aborder un point important qui nous mènera à la troisième partie. Les noms sont placés au plus près du médaillon dans lequel se trouve l'Agneau divin à la voûte de la crypte, ils n'entrent pas dedans, de même qu'aucun nom ne pénètre dans le nimbe d'un saint. ${ }^{43}$ Il semble devoir rester à la frontière, ce qui permet justement le contact entre les deux, un toucher qui est comme un corps à corps.

\section{Incorporer}

Après la coupure vient la soudure. Le dernier verbe de cette trilogie est "incorporer", car nous allons nous intéresser au corps des personnages représentés et parce qu'il permet d'une part de montrer, comme en cuisine, à quel point l'élément ajouté (le nom) est intimement uni à l'image, et d'autre part de questionner la nouvelle unité créée.

Si ce dispositif permet de placer le nom dans une intime proximité avec le corps de la figure, les points de contact entre les deux sont variés : la tête est le plus habituel et regroupe $74 \%$ des occurrences (que le nom soit placé en haut de la tête, au niveau du visage ou du cou,

\footnotetext{
${ }^{42}$ CIFM I-1, 18, pp. 17-18, pl. XII, fig. 20-22 ; CIFM Hors-Série II, 52 (à paraître).

${ }^{43}$ Dans d'autres mises en scènes, au contraire, le nom est volontairement placé sur l'auréole. Pour ne donner qu'un exemple : sur les chapiteaux du rond-point de l'église Saint-Pierre de Chauvigny, Marie porte à plusieurs reprises son nom inscrit sur son nimbe : CIFM I-2, 18, pp. 25-26, pl. XI-XII, fig. 25-27.
} 
par exemple les noms de saint Martin et saint Calmine sur la châsse émaillée de saint Calmine réalisée au XIII ${ }^{\mathrm{e}}$ s. et conservée au Musée Dobrée à Nantes) $;^{44}$ on trouve aussi des noms placés au niveau du tronc dans $15 \%$ des cas (épaules ou milieu du corps) et quelques exemples $6 \%$ dans la moitié inférieure du corps (notamment les genoux) par exemple sur une autre châsse de saint Calmine de la fin du XII ${ }^{\mathrm{e}}$ s. (noms des apôtres Matthieu, Thomas et Pierre), dans l'église Saint-Pierre de Mozac (Puy-de-Dôme). ${ }^{45}$

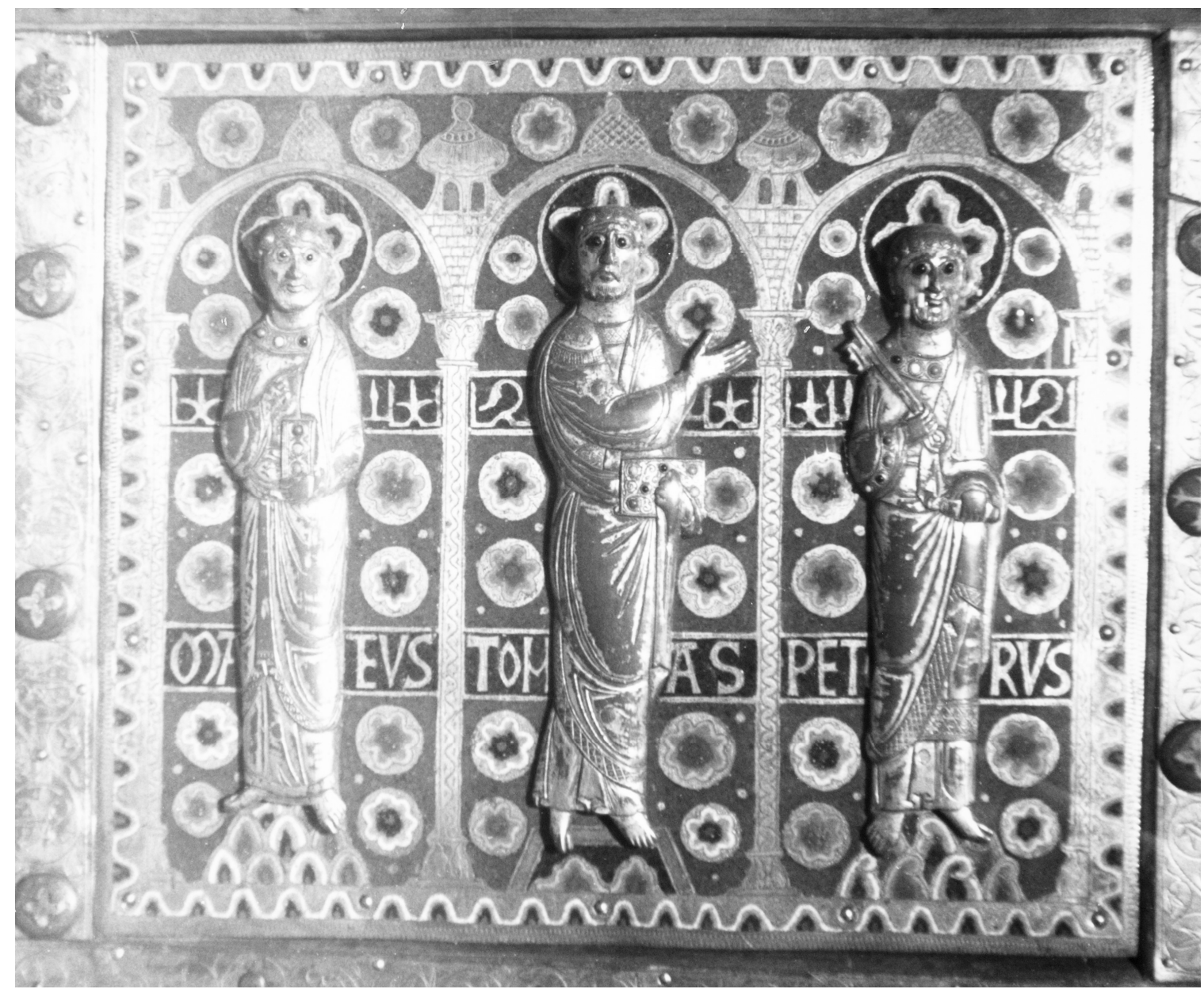

[Figure 8 : châsse de saint Calmine, noms des apôtres Matthieu, Thomas et Pierre, fin du XII ${ }^{\mathrm{e}}$ s., église SaintPierre de Mozac (Puy-de-Dôme). Cliché : Jean Michaud/CIFM-CESCM.]

Les 5\% restant concernent soit des césures dont on ne connaît pas l'emplacement, soit un emplacement plus général prenant l'ensemble de la figure. C'est pourquoi le verbe "flanquer" n'est pas si adapté pour parler de ce dispositif, car si le nom est bien sur les côtés du personnage, il n'est pas principalement au niveau de ses flancs.

La position du texte est importante pour le regardeur. Le visage est considéré comme le miroir de l'âme, le siège de l'identité, porteur des organes sensoriels comme les yeux et la bouche qui projettent le regard et la voix. Un tel emplacement du nom peut donner l'impression que c'est le personnage qui parle, disant : c'est moi, je suis celui dont le nom est écrit, et qu'à son tour le fidèle répond : c'est lui dont je vois le nom. ${ }^{46}$ Le nom joue alors

\footnotetext{
${ }^{44}$ CIFM 23, 70, pp. 82-83.

${ }^{45}$ CIFM 18, Puy-de-Dôme 74, pp. 224-228, pl. CXX-CXXVI, fig. 257-270.

46 Gilbert Dagron propose des remarques similaires au sujet des noms sur les icônes byzantines. Voir: G. DAGRON, Décrire et peindre. Essai sur le portrait iconique (Paris, 2007), pp. 68-69.
} 
pleinement son rôle d'index, ${ }^{47}$ et dans le face à face qui s'instaure entre la représentation et le spectateur, il devient dialogue et fait entendre à la fois la voix du saint et celle du chrétien en prière qui l'implore. La corporéité de la figure est mise en avant dans cette mise en scène.

Le point de contact est certes localisé, le nom n'en étreint pas moins le corps dans son ensemble et semble "incorporer" ainsi le personnage représenté. C'est comme si ce dernier était tout entier dans son nom. Dans la pensée médiévale, le nom a une épaisseur, une profondeur ; la symbolique du nom propre joue un rôle important - particulièrement dans la littérature et l'hagiographie, mais pas seulement ${ }^{48}$ - car celui-ci dit la vérité de la personne. Le nom entretient des rapports étroits avec celui qui le porte et donne sens à la vie ; connaître l'origine d'un nom propre, comprendre son étymologie, c'est souvent connaître la nature profonde de l'être. L'annominatio rapprochant nomen et omen, présente dès l'Antiquité, synthétise cela en insistant sur la réalisation de l'être dans le temps: le nom est présage, augure, il dit l'avenir.

Ces réflexions nous amènent à proposer une double hypothèse : c'est la notion d'être qui est au centre de ce dispositif. Il dépasse la simple identification et même l'identité, pour toucher à l'être profond. On peut alors parler de dispositif ontologique. En ce sens, on peut y voir une traduction ou une matérialisation de la pensée étymologique. Pour Isidore de Séville, héritier d'une double tradition hellénistique et sémitique par la Bible, l'étymologie est une herméneutique de la réalité, un moyen d'investigation plus qu'une science prise pour ellemême. Elle mène de la linguistique à l'ontologie, du mot à l'être. ${ }^{49}$ Cela décrit précisément la fonction graphique, syntaxique et symbolique de ce dispositif. Dans la méthode étymologique, le nom est "ouvert" et divisé en différentes parties pour comprendre ce qui est à l'intérieur ; le même processus a lieu ici.

S'il peut y avoir incorporation, c'est d'une part parce que les deux langages du mot et de l'image relèvent d'une même nature graphique, picturale et gestuelle, d'autre part parce qu'ils partagent la même surface. ${ }^{50}$ La suite horizontale des lettres rencontre la représentation d'un personnage placé à la verticale. Par leur orientation différente, les deux lignes se croisent perpendiculairement. Les deux langages fusionnent et une nouvelle unité iconique naît de cette intersection, ne distinguant plus le personnage de son nom et formant une croix, comme avec Jean dans les peintures murales de Novacelles (Puy-de-Dôme, c. 1300). ${ }^{51}$

\footnotetext{
47 Sur le nom comme énonciation indicielle, voir L'écriture du nom propre, p. 8.

${ }^{48}$ Voir par exemple : M. PASTOUREAU, "Le nom mis en images : les armoiries parlantes (XII ${ }^{\mathrm{e}}-\mathrm{XVII}{ }^{\mathrm{e}}$ siècle)", in Lecture, représentation et citation. L'image comme texte et l'image comme signe (XI ${ }^{e}-X V I I^{e}$ siècle), ed. C. HECK (Lille, 2007), pp. 69-115 ; B. GREVIN, "L'étymologie en action? Questions sur la pratique des annominationes de noms propres dans la rhétorique politique du XIII siècle", Le pouvoir des mots au Moyen Âge, ed. N. BERIOU et al. (Turnhout, 2014), pp. 107-126.

49 Voir G. DAHAN, "Nommer les êtres : exégèse et théories du langage dans les commentaires médiévaux de "Genèse" 2, 19-20", Sprachtheorien in Spätantike und Mittelalter (Tübingen, 1995), pp. 55-74. Sur Isidore et le Moyen Âge, voir le volume 16 des Cahiers de Recherches Médiévales et Humanistes, paru en 2008, intitulé "La réception d'Isidore de Séville durant le Moyen Âge tardif ( $\mathrm{II}^{\mathrm{e}}-\mathrm{XV}^{\mathrm{e}} \mathrm{s}$.)", sous la direction de Jacques Elfassi et Bernard Ribémont.

${ }^{50}$ Sur les questions de surface, voir L'écriture entre support et surface, ed. M. ARABYAN (Paris, 2005), particulièrement la conclusion de ce volume par J. FONTANILLE, "Du support matériel au support formel”, pp. 183-200.

${ }^{51}$ CIFM 18, Puy-de-Dôme 76, p. 230, pl. CXXVIII, fig. 274.
} 


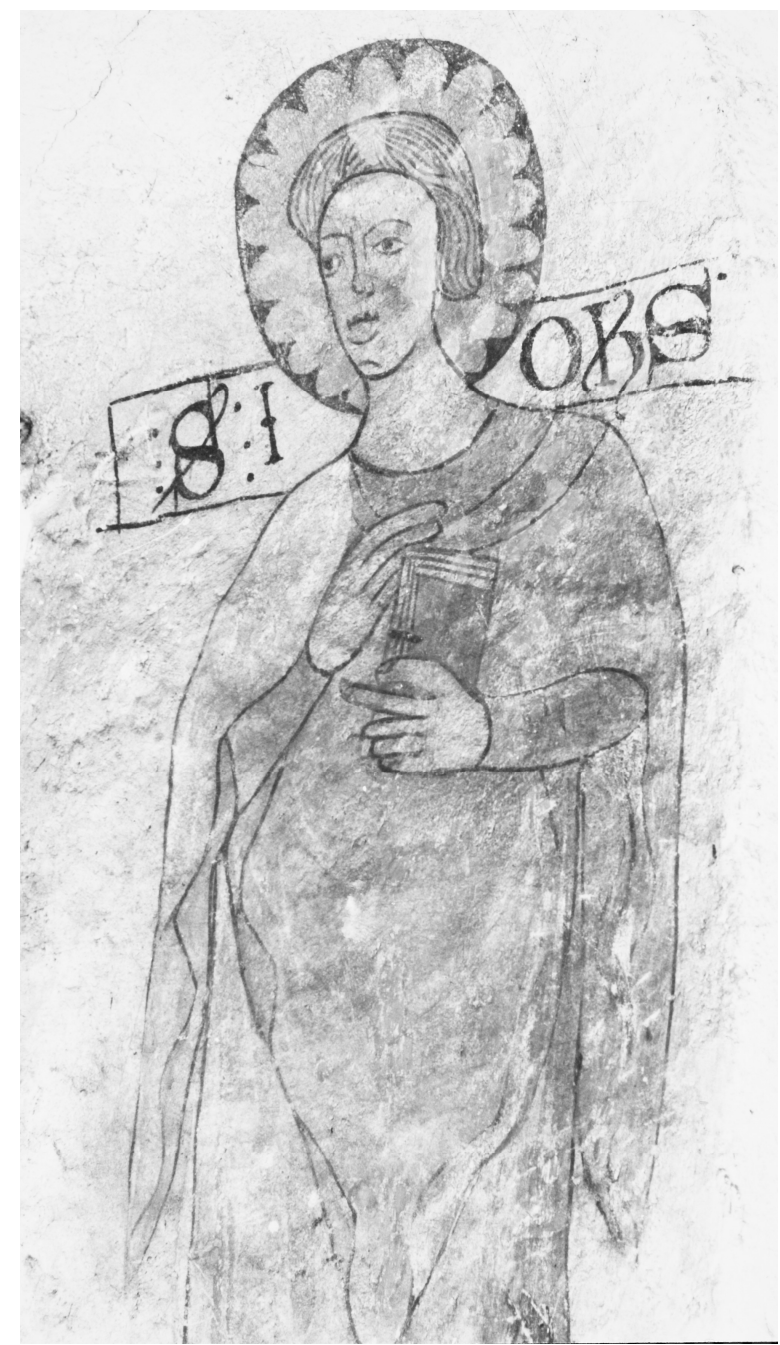

[Figure 9 : Peintures murales de l'abside, c. 1300, (Puy-de-Dôme), église Saint-Pierre de Novacelles. Cliché : Jean Michaud/CIFM-CESCM.]

Dans ce cas précis, le fait que l'inscription soit dans un champ épigraphique clairement délimité, formant un bandeau, renforce l'impression cruciforme de la nouvelle unité. C'est paradoxalement d'une division que naît l'union.

\section{Conclusion :}

Cette courte étude a prêté son attention à une catégorie de mots : les noms, à travers le dispositif ancien de la césure que le Moyen Âge renouvelle, car dans la pensée chrétienne l'acte de nommer tient une place essentielle, il fait exister, depuis l'un des événements linguistiques fondateurs, celui de la Genèse (II, 19-20). Dès lors, quel meilleur mode opératoire pour manifester l'être par le langage, l'écriture et la matière, qu'en les fusionnant? Au-delà d'un simple processus didactique de donner le nom pour reconnaître un personnage, la césure graphique permet, par le croisement entre la trajectoire horizontale des lettres et la figure verticale, un contact et une double dynamique. L'image pénètre le nom, tandis que le mot étreint voire incorpore la représentation, créant une nouvelle unité iconique. Suivant la même méthode qui guide la recherche étymologique, le corps graphique du nom est ouvert pour atteindre l'être même. L'acte de nomination trouve ici une place privilégiée grâce à un condensé sémiotique. 